\title{
Safety of adipose-derived cell (stromal vascular fraction - SVF) augmentation for surgical breast reconstruction in cancer patients
}

\author{
Sławomir Mazur ${ }^{1, A-C, F}$, Aleksandra Zołocińska2,C,D, Katarzyna Siennicka2,C,D, \\ Karolina Janik-Kosacka ${ }^{2, C, D}$, Anna Chrapusta ${ }^{3, C, E}$, Zygmunt Pojda ${ }^{2, A, D, F}$ \\ 1 Department of Breast Cancer and Reconstructive Surgery, Maria Skłodowska-Curie Memorial Cancer Center and Institute of Oncology, Warszawa, Poland \\ 2 Department of Regenerative Medicine, Maria Skłodowska-Curie Memorial Cancer Center and Institute of Oncology, Warszawa, Poland \\ ${ }^{3}$ Department of Plastic and Reconstructive Surgery, Ludwik Rydygier Memorial Specialized Hospital in Kraków, Poland \\ A - research concept and design; $B$ - collection and/or assembly of data; $C$ - data analysis and interpretation; \\ $D$ - writing the article; $E$ - critical revision of the article; $F$ - final approval of the article
}

Address for correspondence

Zygmunt Pojda

E-mail:zpojda@coi.waw.pl

Funding sources

The project was supported by a research grant from the National Centre for Research and Development, Poland (STRATEGMED 1/233224/10/ N(BR/2014).

\section{Conflict of interest}

None declared

Received on 0ctober 27, 2016

Reviewed on March 12, 2017

Accepted on April 27, 2017
DOI

10.17219/acem/70798

Copyright

Copyright by Author(s)

This is an article distributed under the terms of the

Creative Commons Attribution Non-Commercial License

(http://creativecommons.org/licenses/by-nc-nd/4.0/)

\begin{abstract}
Background. Progress in breast cancer surgery results in a decreased frequency of mastectomy, in the early phases of cancer replaced by breast conserving therapy (lumpectomy). Increased popularity of breast reconstruction by fat or adipose stem cells (ASC)-enriched fat transfer raised uncertainty about the possible risk of increased cancer recurrence. In vitro studies suggest that locally secreted cytokines and reconstructed local blood vessels may stimulate cancer expansion or cancer de novo induction from glandular tissue remaining after lumpectomy.
\end{abstract}

Objectives. The purpose of the study was to evaluate the risk of cancer recurrence in breast cancer patients related to the stromal vascular fraction (SVF) augmentation during autologous fat grafting for breast reconstruction.

Material and methods. The tumor recurrence ratio in 56 patients having the breast reconstructed with autologous ASC (transplanted as the subpopulation present in SVF) was compared with the frequency of tumor recurrence in 252 matched patients treated in clinics without subsequent breast reconstruction. Adipose tissue was collected by the Coleman technique and split into 2 portions: one was used for breast reconstruction, the other was enzymatically digested, and isolated cells were used for the augmentation of fat implanted into the breast area. Cancer recurrence in the experimental and matched control group was evaluated following 3-year-long observation time, and the statistical significance of difference in cancer recurrence between the experimental and control group was evaluated.

Results. Cancer recurrence in the group of patients treated with ASC-enriched fat for breast reconstruction was $3.7 \%$ and did not differ significantly from the control group data (4.13\%). No adverse effects of therapy were observed.

Conclusions. Our study does not produce any data suggesting increased cancer risk following breast reconstruction after a mastectomy or a lumpectomy combined with local radiotherapy. It may be concluded that an autologous transplantation of fat augmented with ASC is a safe and efficient procedure. Longer observation time and the observation of larger numbers of patients would be useful for strengthening the conclusion.

Key words: mesenchymal stem cells, adipose tissue, treatment-associated cancer 


\section{Introduction}

The purpose of the study was to evaluate the risk of cancer recurrence in breast cancer patients related to the stromal vascular fraction (SVF) augmentation during autologous fat grafting for breast reconstruction.

Breast cancer treatment results in permanent tissue injuries in the region of cancer localization. The problem is not restricted only to esthetic aspects; surgery and local radiotherapy produce injuries of connective tissue, blood and lymphatic vessels, or nerves, resulting in pain, lymphedema and deformations. The method of autologous fat grafting is growing in popularity, as it is more physiological than the implantation of artificial substances like silicone implants or injectable fillers. The disadvantage of the lipofilling technique is fat resorption ranging from $25 \%$ to $80 \%$.

Adipose stem cells (ASC), when added to transplanted fat, may differentiate into new adipocytes, thus preserving more permanently the volume of fat used for breast reconstruction. When adding the mixed cell population (stromal vascular fraction - SVF) obtained as a result of enzymatic fat digestion, such cells as endothelial progenitor cells (EPC) may strengthen the effect of ASC on neoangiogenesis, increasing the survival of transplanted adipocytes. Immune system cells, also present in SVF, prevent infections immediately after fat grafting, before new blood vessels colonize transplanted adipose tissue. The antiapoptotic role of ASC, their role in tissue repair and adipogenesis as well as the angiogenetic role of EPC and infection prevention by immune cells are strong arguments supporting the use of SVF for breast conserving therapy. ${ }^{1,2}$ There is, however, a not fully clarified problem concerning the possible risk of cancer recurrence induced by ASC. Adipose tissue, even when not supplemented with ASC, contains approx. $3 \times 10^{5} / \mathrm{mL}$ mesenchymal stem cells (MSC), which in in vitro and in animal experiments may stimulate cancer cells. ${ }^{3}$

The problem of the supportive activity of MSC for cancer recurrence, expansion or metastases has been widely researched. All supportive evidence suggesting the stimulatory role of MSC in cancerogenesis originates from in vitro studies and, less numerous, animal

Table 1. Characteristics of patients in respect to the histological type of disease

\begin{tabular}{|l|c|c|c|c|}
\hline $\begin{array}{c}\text { Histological } \\
\text { type }\end{array}$ & $\begin{array}{c}\text { Number } \\
\text { of patients }\end{array}$ & Age [years] & $\begin{array}{c}\text { Transplanted fat } \\
\text { volume [mL] }\end{array}$ & $\begin{array}{c}\text { Number } \\
\text { of transplanted } \\
\text { cells [x106] }\end{array}$ \\
\hline Cdis & $4 / 56(7.1 \%)$ & $46 \pm 10.2$ & $65.2 \pm 17.5$ & $68.9 \pm 56.2$ \\
\hline Cdis, Nst & $1 / 56(1.8 \%)$ & 55 & N/A & 41.0 \\
\hline Fibrosa & $1 / 56(1.8 \%)$ & 73 & 20.0 & 50.8 \\
\hline Lobular & $5 / 56(8.9 \%)$ & $49.4 \pm 7.6$ & $75.3 \pm 19.3$ & $51.4 \pm 22.9$ \\
\hline Nst & $40 / 56(71.4 \%)$ & $49.2 \pm 10.1$ & $88.9 \pm 31.9$ & $85.4 \pm 73.0$ \\
\hline Tubular & $5 / 56(8.9 \%)$ & $52.6 \pm 11.5$ & $69.6 \pm 32.5$ & $91.5 \pm 68.8$ \\
\hline Average & - & $49.8 \pm 10.2$ & $82.9 \pm 31.7$ & $80.3 \pm 67.3$ \\
\hline
\end{tabular}

Cdis - carcinoma ductale in situ; Nst - invasive carcinoma of no special type; N/A - not available. experiments. ${ }^{4}$ To our knowledge, no clinical evidence of oncostimulatory activity has been reported from clinical trials, and the evidence from clinical observations suggests no increase of cancer recurrence. The relatively low number of clinical observations of the MSC - cancer interrelations and the low number of patients included in the studies make it necessary to collect new data before formulating a final conclusion regarding the safety of MSC therapies in patients cured from cancers.

\section{Material and methods}

The Bioethics Committee of the Polish Ministry of Health approved the clinical experiment based on the transplantation of autologous fat augmented with ASC into patients cured from breast cancer (approval No. OKB-4/12).

\section{Patients}

A total number of 56 patients who had undergone breast cancer treatment were enrolled in the study. The control group was matched among patients treated for breast cancer in the years 2012-2015 in Maria Skłodowska-Curie Memorial Cancer Center and Institute of Oncology in Warszawa, Poland.

The inclusion criteria were: the age of 18-75 years, completely cured breast cancer, min 12 months after the completion of treatment, no syndrome of cancer recurrence, and min $2 \mathrm{~mm}$ of fat tissue between the breast skin and the chest wall. The exclusion criteria were: cancer recurrence during the last 12 months, the coexistence of different cancer types, autoimmunological diseases, keloid, connective tissue diseases, chronic anticoagulants treatment ( $<15$ days before the therapy), body mass index (BMI) $>30$, contraindications for magnetic resonance imaging (MRI), expected survival time $<12$ months, and family predispositions for breast cancer, including $B R C A 1$ and $B R C A 2$ gene carriers. The summary of the enrolled patients' characteristics is shown in Tables 1-3. Data on breast reconstruction using autologous fat augmented with autologous ASC of 56 patients was compared with the results of 252 control group patients. The control group included patients of matched age, year of admission to the clinic, histological type of cancer, and treatment protocol - the only difference was the lack of breast reconstruction treatment.

\section{SVF isolation}

All procedures were performed under general anesthesia according to Coleman method. ${ }^{5}$ Prior to liposuction, $20-30 \mathrm{~mL}$ of sterile saline solution supplemented with epinephrine and lignocaine was evenly distributed 
into the collection site by needle injection. Subsequently, $200 \mathrm{~mL}$ of fat tissue was aspirated into $20 \mathrm{~mL}$ syringes. The collected lipoaspirate was divided into 2 portions: one was used for breast reconstruction, the other was enzymatically digested as we described before to isolate SVF cells. ${ }^{6}$ Briefly, phosphate-buffered solution (PBS) (Life Technologies, Waltham, USA)-diluted fat tissue was incubated with $0.025 \%$ collagenase from Clostridium histolyticum (Sigma-Aldrich; St Louis, USA) at $37^{\circ} \mathrm{C}$ for $1.5 \mathrm{~h}$. The resulting mixture was diluted in $\mathrm{PBS}+2 \%$ human albumin (Kedrion Biophrama, Barga, Italy) and centrifuged (400 g, 10 min). Following phase separation, the cell pellet was resuspended in physiological salt solution for patient injections $+5 \%$ human albumin.

\section{Cells analysis}

The number (Burker's chamber and the Sysmex analysis) and viability (acridine orange + ethidium bromide staining, fluorescence microscopy) of cells were determined and sterility control was carried out for every sample (BACTEC, Bact/Alert; Becton Dickinson, Franklin Lakes, USA; blood agar and BHI agar). The cytometric analysis of the SVF cells and adherent fraction cells (ASC) phenotype was performed for the presence of surface markers (CD29, CD31, CD34, CD45, CD73, CD90, CD105, Lin1, CXCR4, and HLA-DR). In vitro proliferation potential (doubling time), clonality (the colony-forming unit (CFU) test) and capability to differentiate into osteogenic, chondrogenic and adipogenic lineage were analyzed in order to confirm the MSC character of the cells (Fig. 1).

\section{SVF application}

The SVF cells were resuspended in physiological salt solution and were used for the augmentation of fat implanted into the breast area in an average amount of $8.4 \times 10^{5}$ cells for every $1 \mathrm{~mL}$ of fat tissue transplanted earlier. The time between fat and cell injections did not exceed $4 \mathrm{~h}$.

\section{Statistical analysis}

Cancer recurrence in experimental vs control group was evaluated using the Fisher's exact test; $\mathrm{p}<0.05$ was considered statistically significant. The data was presented as the average values and standard deviations (SD).

\section{Results}

In patients treated with ASCaugmented adipose tissue, the histological classification of cancer type
(Table 1) revealed the prevalence of invasive carcinoma of no special type (40 patients), 5 cases of tubular carcinoma, 5 cases of lobular carcinoma, 4 cases of carcinoma ductale in situ, and single cases of ductale/no special type and fibrosa. Both cases of cancer recurrence were of invasive no special type, similarly as 10 recurrences in the control group. The number of patients in respect to molecular subtype of cancer, tumor size and the number of lymph nodes involved is presented in Table 2. As reflected in Table 3, most patients were treated by surgical mastectomy combined with radiotherapy (21), mastectomy alone (6), mastectomy combined with implant (9), lumpectomy (7), and transverse rectus abdominis myocutaneous (TRAM) (9). Total reconstruction was performed in 1 patient, rarely ulceration (1) and lymphatic edema (1) were observed. The average volume of transplanted fat varied

Table 2. Characteristics of patients in respect to histological/molecular subtype, tumor size and the number of lymph nodes involved

\begin{tabular}{|l|c|c|c|}
\hline \multicolumn{1}{|c|}{$\begin{array}{c}\text { Histological type/ } \\
\text { molecular subtype }\end{array}$} & $\begin{array}{c}\text { Number } \\
\text { of patients }\end{array}$ & $\begin{array}{c}\text { Tumor size } \\
{[\mathrm{mm}]}\end{array}$ & $\begin{array}{c}\text { Number } \\
\text { of involved } \\
\text { lymph nodes }\end{array}$ \\
\hline Cdis & $4 / 56(7.1 \%)$ & $34.5 \pm 25.8$ & 0 \\
\hline Cdis, Nst/Luminal A & $1 / 56(1.8 \%)$ & 60 & 0 \\
\hline Fibrosa/ - & $1 / 56(1.8 \%)$ & 40.0 & 0 \\
\hline Lobular/Lum B (Her2-) & $2 / 56(3.6 \%)$ & $28.0 \pm 4.2$ & $1.0 \pm 1.4$ \\
\hline Lobular/Luminal A & $2 / 56(3.6 \%)$ & $33.5 \pm 9.2$ & 0 \\
\hline Lobular NoLum/Her2+ & $1 / 56(1.8 \%)$ & 31.0 & 4 \\
\hline Nst/Lum B (Her2-) & $7 / 56(12.5 \%)$ & $32.0 \pm 2.7$ & $1.1 \pm 0.9$ \\
\hline Nst/Lum B (Her2+) & $4 / 56(7.1 \%)$ & $33.5 \pm 2.7$ & $3.5 \pm 0.6$ \\
\hline Nst/Luminal A & $22 / 56(39.3 \%)$ & $34.8 \pm 3.9$ & $0.5 \pm 0.5$ \\
\hline Nst/Non Luminal & $3 / 56(5.4 \%)$ & $35.3 \pm 0.6$ & $5.3 \pm 1.5$ \\
\hline Nst/Triple neg & $4 / 56(7.1 \%)$ & $28.5 \pm 5.1$ & $3.0 \pm 1.8$ \\
\hline Tubular/Luminal A & $5 / 56(8.9 \%)$ & $13.0 \pm 3.4$ & 0 \\
\hline
\end{tabular}

Cdis - carcinoma ductale in situ; Nst - invasive carcinoma of no special type; Lum - luminal; NoLum - non luminal; neg - negative.
Table 3. Characteristics of patients in respect to the treatment protocol

\begin{tabular}{|l|c|c|c|c|}
\hline Treatment protocol & $\begin{array}{c}\text { Number } \\
\text { of patients }\end{array}$ & Age [years] & $\begin{array}{c}\text { Transplanted fat } \\
\text { volume [mL] }\end{array}$ & $\begin{array}{c}\text { Number } \\
\text { of transplanted } \\
\left.\text { cells [x10 } 0^{6}\right]\end{array}$ \\
\hline BCT & $7 / 56(12.5 \%)$ & $50.7 \pm 7.2$ & $78.5 \pm 37.4$ & $102.8 \pm 74.3$ \\
\hline Implant & $9 / 56(16.1 \%)$ & $52.0 \pm 10.3$ & $80.1 \pm 24.6$ & $67.9 \pm 41.0$ \\
\hline M & $6 / 56(10.7 \%)$ & $40.3 \pm 11.1$ & $79.8 \pm 26.6$ & $97.3 \pm 37.9$ \\
\hline M+RT & $21 / 56(37.5 \%)$ & $50.1 \pm 10.2$ & $82.4 \pm 32.0$ & $76.5 \pm 87.2$ \\
\hline Lymphatic edema & $1 / 56(1.8 \%)$ & 61.0 & 108.0 & 36.0 \\
\hline Ulceration & $2 / 56(3.6 \%)$ & $67.5 \pm 7.7$ & $23.5 \pm 4.9$ & $59.4 \pm 12.1$ \\
\hline Total reconstruction & $1 / 56(1.8 \%)$ & 43.0 & 141.0 & 49.7 \\
\hline TRAM & $9 / 56(16 \%)$ & $48.2 \pm 7.3$ & $96.2 \pm 23.7$ & $85.7 \pm 48.8$ \\
\hline Average & - & $49.8 \pm 10.2$ & $82.9 \pm 31.6$ & $80.3 \pm 67.3$ \\
\hline
\end{tabular}

$\mathrm{BCT}$ - breast conserving therapy; $\mathrm{M}$ - mastectomy; $\mathrm{M}+\mathrm{RT}$ - mastectomy and radiotherapy; TRAM - transverse rectus abdominis myocutaneous. 
A

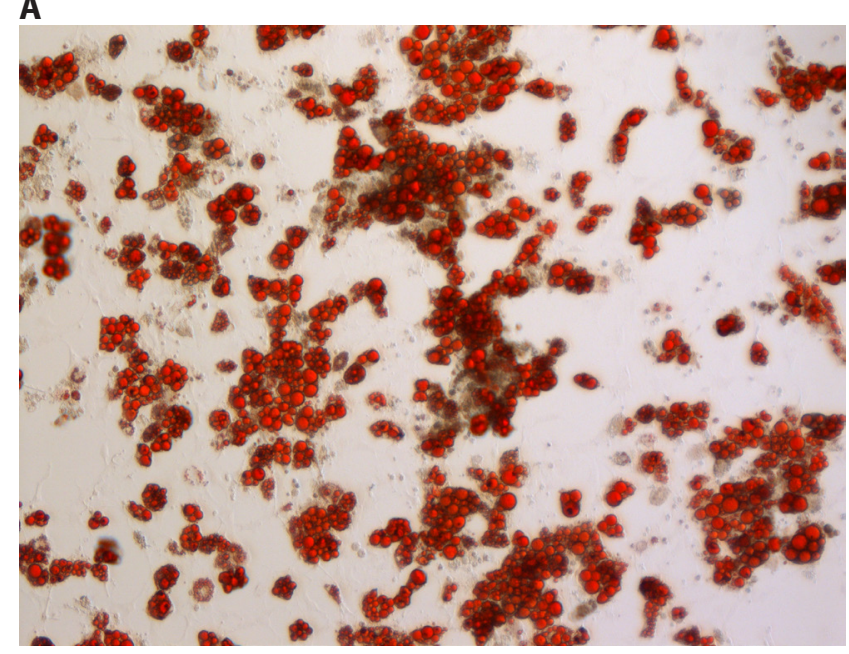

B

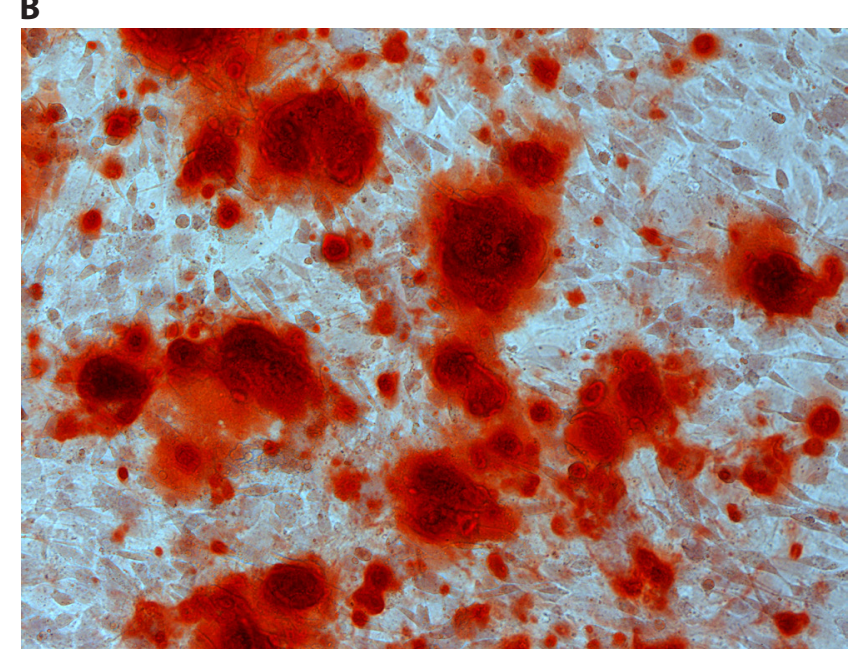

C

Fig. 1. Differentiation of adipose stem cells (ASC) into adipogenic (a), osteogenic (b) and chondrogenic (c) lineages

The cells were treated by differentiation media (all from Lonza, Allendale, USA) according to the manufacturer's protocol, and stained by Oil Red O for adipogenic differentiation, Alizarin Red for osteogenic differentiation and Messon's Trichrome for chondrogenic differentiation (Sigma-Aldrich, St. Louis, USA). from $23.5 \mathrm{~mL}$ (ulceration treatment) up to $141 \mathrm{~mL}$ (total reconstruction). The average number of the SVF cells transplanted varied between $36 \times 10^{6}$ (lymphatic edema) and $102.8 \times 10^{6}$ (breast conserving therapy) (Table 3 ). Locoregional cancer recurrence in the experimental group in patients with invasive no special type carcinoma was $2 / 54$ and in the matched control group it was $10 / 242$, which constituted $3.70 \%$ and $4.13 \%$, respectively. The difference between cancer recurrence in the experimental vs control group was statistically insignificant (the Fisher's exact test, $\mathrm{p}=1.0)$.

\section{Discussion}

Mesenchymal stem cells are present in all the tissues of the human body as the cellular component of connective tissue. Their capacities, like a regulatory role in tissue repair, an immunomodulatory role in suppressing immune reactions against own and transplanted allogeneic tissues, and differentiation into several tissues make them prime candidates for regeneration medicine treatments. Their medical applications are, however, at the stage of in vivo tests or clinical trials, none of them being utilized in regular clinical procedures. They are most frequently tested in orthopedics and plastic surgery, and also in neurology, cardiology or transplantology.

Mesenchymal stem cells maybe collected from adult donors, which makes it possible to use them for autologous purposes. The source containing the highest number of MSC, which may be easily collected, is adipose tissue (adipose stem cells - ASC). These cells fulfill the morphologic and functional criteria listed by the International Society for Cellular Therapy Committee as mandatory for mesenchymal stem (stromal) cells. ${ }^{7,8}$ One of these criteria is differentiation into adipose tissue, which is the main reason for the application of ASC for the supplementation of fat used for breast reconstruction in oncological patients. The other ASC capability when transplanted into fat-reconstructed breast

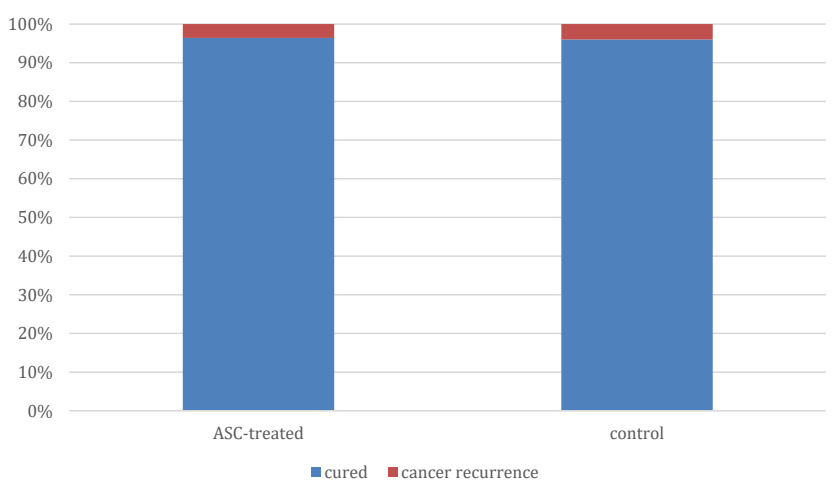

Fig. 2. Comparison of the cancer recurrence rate in the experimental (SVF-enriched fat lipofilling) and control group of patients ASC - adipose stem cells; SVF - stromal vascular fraction. 
is its angiogenic potential resulting in the production of blood vessels, and preventing the apoptosis or necrosis of transplanted adipocytes. The beneficial effects of the ASC enrichment of fat used for breast reconstruction have been experimentally confirmed. Matsumoto et al. observed that ASC are transplanted with vascular endothelial cells and contribute to neoangiogenesis in the acute phase of transplantation. ${ }^{9}$ The unanswered question was how important the transplanted ASC are in the compensation of fat resorption by the production of new adipocytes. The question was solved by the elegant experiment done by Kolle et al., who implanted fat deposits into the upper arms of healthy volunteers. ${ }^{10}$ After 121 days, the fat deposits were removed, their content was histologically analyzed and the volumes of fat deposits, enriched by the ASC addition, were compared with the volumes of fat implanted without the addition of ASC. The volumes of ASC-enriched fat deposits were $80.9 \%$ of the initial volume, whereas fat-only deposits were $16.3 \%$.

A still unsolved dispute concerns the risk of cancer induction by MSC. There is no data available on the possible cancer transformation of the transplanted MSC (up to several months of in vitro culture prior to transplantation). ${ }^{11}$ Animal studies confirmed that even very high numbers (up to $450 \times 10^{6}$ cells per animal) of allogeneic MSC were transplanted without any negative effects. ${ }^{12}$ There is, however, some data from in vitro and, to a lesser extent, animal in vivo experiments (immunocompromised mice with heterotopic cancer) documenting the possibility of cancer stimulation by MSC co-cultured in vitro or injected into animals. ${ }^{13-19}$ In vitro, ASC in co-culture with breast cancer cell lines promote the proliferation, induction of epithelial-to-mesenchymal transition (EMT) (through P13K/AKT signaling in breast cancer cells), enhancement of migration, invasion of breast cancer cells by cell-cell contact, and the secretion of IL- 6 and IL-8. ${ }^{13}$ When adding MSC to 3D-cultured hepatocarcinoma cells, they upregulate the matrix metaloproteinase (MMP), EMT-related genes and increase the migration ability, thus enhancing the tumor metastasis ability. ${ }^{14}$

Bone marrow MSC, mixed with weakly metastatic human breast carcinoma cells, increased their metastatic potency, secreting de novo CCL5 chemokine (RANTES). ${ }^{15}$ Adipose stem cells of phenotype $\left(\mathrm{CD} 45^{+}, \mathrm{CD} 34^{+}, \mathrm{CD}^{2} 1^{+}\right.$, $\mathrm{CD}_{13}{ }^{+}, \mathrm{CD}_{140 \mathrm{~b}^{+}}$) generated pericytes and were efficient in promoting local tumor growth, inducing EMT gene expression in luminal breast cancer cells. ${ }^{16}$ In in vivo experiments in immunocompromised mice, the coinjection of human ASC from lipotransfer contributed to tumor vascularization and significantly increased tumor growth and metastases in orthotopic models of human breast cancer. ${ }^{17,18}$ Eterno et al. reported that ASC are not tumorigenic per se and are not able to induce the metastatic transformation of normal mammary cells, and that breast cancer recurrence and the enhancement of tumor cell migration and tumor cell renewal resulted from the HGF/cMet crosstalk between ASC and breast cancer cells. ${ }^{19}$
Surprisingly, this data is not reflected by the observations collected from clinical trials involving patients cured from cancer, treated with autologous or allogeneic MSC. The results of the implantation of ASC-containing grafts into patients treated for osteosarcoma, chondrosarcoma or Ewing sarcoma did not produce any side effects, including cancer recurrence. ${ }^{20} \mathrm{~A}$ multicenter study on breast reconstruction with ASC-enriched fat also did not produce breast cancer induction by ASC added to lipofilling material. ${ }^{21}$ The authors of a recently published study based on the analysis of extensive literature data conclude that "recent reports concerning SVF/ASC enrichment of fat graft did not describe any significant worsening of prognosis for patients; however, further studies and longer follow-ups are needed to confirm the safety of procedures using SVF/ASC enrichment during post-surgical breast reconstruction". ${ }^{22}$ Likewise, the authors of a similar analysis of published data conclude: "If the family history is negative for breast cancer and no additional risk factors for breast cancer are evident, fat grafting can be considered a surgical option." 23 The difference between the in vitro and in vivo (mice) studies and clinical data may be explained by the fact that both in vitro research and animal studies are very simplified when compared to the clinical situation. Such an experimental design does not allow for the immune system involvement, and tests ASC in an artificial environment, which may change their secretome production. In contrast, enriching adipose tissue with ASC makes only a quantitative, but not qualitative, difference, since non-enriched fat also contains a relatively high number of ASC and the ASC additionally admixed into fat are placed in their "native" environment. There are also scant reports on the anticancer activity of ASC. Human MSC in a hepatoma model inhibit the malignant phenotypes of human liver cancer lines, which includes the proliferation, colony-forming ability and oncogene expression, both in vitro and in vivo. ${ }^{24}$ Otsu et al. observed that in an in vivo tumor model, direct MSC inoculation at high numbers into subcutaneous melanomas induced apoptosis and abrogated tumor growth due to their antiangiogenic mechanism. ${ }^{25}$ Similarly, the anticancer activity of marrow MSC was observed in an experimental model of Kaposi sarcoma, and in an in vitro or in vivo model when ASC induced pancreatic cancer death. ${ }^{26,27}$

The clinical benefits of breast reconstruction were not analyzed due to the relatively low number of patients and a high heterogeneity of cancer types, disease stages and cancer therapy protocols. The clinical outcome (residual pain, lymphatic edemas) and their satisfaction levels were better in the breast reconstruction group. Our study was concentrated on the evaluation of the safety of the SVF treatment in patients cured from cancer. Our observation time was relatively short, hence the conclusions must be considered with caution. However, in other papers, the follow-up period was 12 months, 14-48 months vs our 24-36-month follow-up time..$^{21,28}$ 
It may be concluded that breast reconstruction using fat augmented with autologous adipose-derived stem cells does not increase the risk of cancer recurrence. The relatively short observation time (3 years) and the heterogeneity of both cancer types and cancer treatment protocols suggest a cautious interpretation of our results.

\section{References}

1. Rigotti G, Marchi A, Galie M, et al. Clinical treatment of radiotherapy tissue damage by lipoaspirate transplant: A healing process mediated by adipose-derived adult stem cells. Plast Reconstr Surg. 2007;119:1409-1422.

2. Ryden M, Dicker A, Gotherstrom C, et al. Functional characterization of human mesenchymal stem cell-derived adipocytes. Biochem Biophys Res Commun. 2003;311:391-397.

3. Muehlberg FL, Song $\mathrm{YH}$, Krohn A, et al. Tissue-resident stem cells promote breast cancer growth and metastasis. Carcinogenesis. 2009;30:589-597.

4. Zimmerlin L, Donnenberg AD, Rubin JP, Basse P, Landreneau RJ, Donnenberg VS. Regenerative therapy and cancer: In vitro and in vivo studies of the interaction between adipose-derived stem cells and breast cancer cells from clinical isolates. Tissue Eng Part A. 2011;17:93-106.

5. Coleman SR. Facial recontouring with lipostructure. Cain Plast Surg. 1997;24(2):347-367.

6. Siennicka K, Zołoćińska A, Stępień K, et al. Adipose-derived cells (stromal vascular fraction) transplanted for orthopedical or neurological purposes: Are they safe enough? Stem Cells Int. 2016; 2016. doi.10.1155/2016/5762916

7. Dominici M, Le Blanc K, Mueller I, et al. Minimal criteria for defining multipotent mesenchymal stromal cells: The International Society for Cellular Therapy position statement. Cytotherapy. 2006;8:315-317.

8. Horwitz EM, Le Blanc K, Dominici M, et al.; International Society for Cellular Therapy. Clarification of the nomenclature for MSC: The International Society for Cellular Therapy position statement. Cytotherapy. 2005;7:393-395.

9. Matsumoto D, Sato K, Gonda K, et al. Cell-assisted lipotransfer: Supportive use of human adipose-derived cells for soft tissue augmentation with lipoinjection. Tissue Eng. 2006;12:3375-3382.

10. Kolle S-FT, Fischer-Nielsen A, Mathiasen AB, et al. Enrichment of autologous fat grafts with ex-vivo expanded adipose tissue-derived stem cells for graft survival: A randomised placebo-controlled trial. Lancet. 2013;382:1113-1120.

11. Rubio D, Garcia-Castro J, Martin MC, et al. Spontaneous human adult stem cell transformation. Cancer Res. 2005;65:3035-3039.

12. McDonald CA, Oehme D, Pham Y, et al. Evaluation of the safety and tolerability of a high-dose intravenous infusion of allogeneic mesenchymal precursor cells. Cytotherapy. 2015;17:1178-1187.

13. Ritter A, Friemel A, Fornoff F, et al. Characterization of adipose-derived stem cells from subcutaneous and visceral adipose tissues and their function in breast cancer cells. Oncotarget. 2015;6:34475-34493.

14. Liu C, Liu Y, Xu XX, Guo X, Sun GW, Ma XJ. Mesenchymal stem cells enhance the metastasis of 3D-cultured hepatocellular carcinoma cells. BMC Cancer. 2016;16:566. doi:10.1186/s12885-016-2595-4

15. Karnoub AE, Dash AB, Vo AP, et al. Mesenchymal stem cells with in tumor stroma promote breast cancer metastasis. Nature. 2007;449:557-563.

16. Orecchioni S, Gregato G, Martin-Padura I, et al. Complementary populations of human adipose CD34+ progenitor cells promote growth, angiogenesis, and metastasis of breast cancer. Cancer Res. 2013;73:5880-5891.

17. Manzotti M, Lohsiriwat V, Rietjens M, Petit JY, Bertolini F. The white adipose tissue used in lipotransfer procedures is a rich reservoir of CD34+ progenitors able to promote cancer progression. Cancer Res. 2012;72:325-334.

18. Kamat $P$, Schweizer $R$, Kaenel $P$, et al. Human adipose-derived mesenchymal stromal cells may promote breast cancer progression and metastatic spread. Plast Reconstr Surg. 2015;136:76-84.

19. Eterno V, Zambelli A, Pavesi L, et al. Adipose-derived mesenchymal stem cells (ASCs) may favour breast cancer recurrence via HGF/c-Met signaling. Oncotarget. 2013;5:613-633.
20. Veriter S, Andre W, Auassar N, et al. Human adipose-derived mesenchymal stem cells in cell therapy: Safety and feasibility in different "hospital exemption" clinical applications. PLoS One. 2015. doi:10.1371/journal.pone.0139566

21. Perez-Cano R, Vrancks JJ, Lasso JM, et al. Prospective trial of adiposederived regenerative cell (ADRC)-enriched fat grafting for partial mastectomy defects: The RESTORE-2 trial. Eur J Surg Oncol. 2012;38:382-389.

22. Bielli A, Scioli MG, Gentile P, Cervelli V, Orlandi A. Adipose tissuederived stem cell therapy for post-surgical breast reconstruction: More light than shadows. Adv Clin Exp Med. 2015;24(3):545-548.

23. Krumboeck A, Giovanoli P, Plock JA. Fat grafting and stem cell enhanced fat grafting to the breast under oncological aspects: Recommendations for patient selection. The Breast. 2013;22:579-584.

24. Qiao L, Xu Z, Zhao T, et al. Suppression of tumorigenesis by human mesenchymal stem cells in a hepatoma model. Cell Res. 2008;18:500-507.

25. Otsu K, Das S, Houser SD, Quadri SK, Bhattacharta S, Bhattacharya J. Concentration-dependent inhibition of angiogenesis by mesenchymal stem cells. Blood. 2009;113:4197-4205.

26. Zhu W, Xu W, Jiang R, et al. Mesenchymal stem cells derived from bone marrow favor tumor cell growth in vivo. Exp Mol Pathol. 2006;80:267-274.

27. Cousin B, Ravet E, Poglio $S$, et al. Adult stromal cells derived from human adipose tissue provoke pancreatic cancer cell death both in vitro and in vivo. PLoS One. 2009;4:e6278.

28. Mestak O, Hromadkova V, Fajfrova M, et al. Evaluation of oncological safety of fat grafting after breast-conserving therapy: A prospective study. Ann Surg Oncol. 2016;23:776-781. 